\title{
Quantum dots photoinduced charges dynamics - model of crystall lattice defects
}

\author{
Artemiy Shmelev*, Victor Nikiforov, Dmitrii Zharkov, Andrey Leontyev, and \\ Vladimir Lobkov \\ Zavoisky Physical-Technical Institute, FRC Kazan Scientific Center of RAS, 420029 Kazan, Russia
}

\begin{abstract}
We report the results of core-shell (CdSe/CdS) quantum dots study. Quantum dots sizes were evaluated as 2.0 and $2.9 \mathrm{~nm}$ from absorbance edge position. We suggest two types of traps, predict properties of these traps based on upconversion luminescence data and previous studies of quantum dots (CdSe cores only) and bulk CdS.
\end{abstract}

Semiconductor quantum dots (QDs) are actively used in science and technology, due to their unique physical and chemical properties [1]. As is known from [2], QDs exhibit a multiexponential luminescence decay with characteristic times depending on their size, up to hundreds of nanoseconds. This work focuses on the analysis of the experimental results of up-conversion spectroscopy of luminescence obtained for QDs of $2 \mathrm{~nm}$ and $2.9 \mathrm{~nm}$ size using data obtained on a CdS single crystal and previously published data.

As is known [3, 4] certain photoexcitation relaxation processes should prevail depending on the size of QDs. At large sizes, it is electron-phonon relaxation that predominates, while at smaller sizes it is Auger recombination. We expected to see a significant difference between the "fast" (subpicosecond) and "slow" (tens of picosecond) contributions for QDs of different sizes. However, we have found by means of luminescence up-conversion spectroscopy [5] that the qualitative form of the luminescence decay does not depend on the size of the QD, which indicates an identical type of relaxation processes for "large" and "small" QDs. Nevertheless, the parameters of the two-exponential decay of the luminescence kinetics depend significantly on the size of the QDs: 1.8 and 26.8 ps correspond to QDs with a size of $2 \mathrm{~nm} ; 4.5$ and 68 ps correspond to QDs with a size of $2.9 \mathrm{~nm}$.

Based on a model from published data [5-9] obtained using incoherent photon echo spectroscopy [10-12], we suggested that the features of the detected luminescence kinetics can be explained as follows: an increase in the luminescence intensity is associated with the population of the bottom of the conduction band during electron termalization. We assume the existence of one type (Fig. 1, in the right) of "shallow" traps (lying shallow relative to the conduction band) and one type of "deep" traps (lying far from the conduction band). A prolonged decrease in the luminescence kinetics should be correlated with the quenching of excited levels due to the population of "deep" traps. We assume that "shallow" traps

\footnotetext{
* Corresponding author: sgartjom@gmail.com
} 
correspond to defects inside the core of the QDs, and "deep" traps correspond to defects at the core-shell interface and further [5].

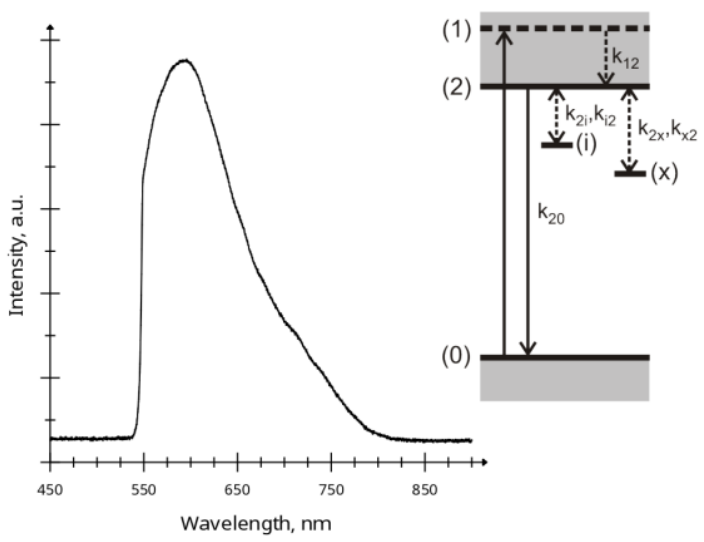

Fig. 1. Luminescence spectra of CdSe cores only QDs on the left, and suggested energy levels on the right $(0$ - valence band, 2 - conducting band, 1 - some energy level in conducting band, i - "shallow" traps, $\mathrm{x}-$ "deep" traps, $\mathrm{k}$ - speed constants).

As a result of using this trap model, we can try to explain spectra of core-only CdSe QDs in Fig. 1. Possible recombination from trap levels is the origin of complex shape of the lower energy tail of CdSe cores QDs spectrum. This spectrum was obtained in confocal setup with $532 \mathrm{~nm}$ laser excitation of CdSe cores only QDs deposited on glass slide.

This work was partially supported by the Russian Foundation for Basic Research, project nos. 18-5200026Bel_a, 17-02-00701a,19-02-00569a and 19-03-00635 (optical measurements, theoretical background), and from the government assignment for FRC Kazan Scientific Center of RAS (sample preparing). The confocal setup measurements were supported by Act 220 of the Russian Government (Agreement No. 14.W03.31.0028 with the host organization ZPTI of KazanSC of RAS).

\section{References}

1. M. Maleki, M. Sasani., Semicond. Phys. Quant. Electr. \& Opt.electr. 10, 30 (2007)

2. U. Resch-Genger et al., Nature Methods. 5, 763 (2008)

3. S.S. Prabhu, A.S. Vengurlekar, J. Shah, Phys. Rev. B. 53, R10465 (1996)

4. V.I. Klimov, D.W. McBranch, Phys. Rev Lett. 80, 4028 (1998)

5. V.G. Nikiforov et al., Las. Phys. Lett. 16, 065901 (2019)

6. K.R. Karimullin et al., J. Phys. Conf. Ser. 859, 012010 (2017)

7. A.I. Arzhanov et al., Bull. Lebedev Phys. Inst. 45, 91 (2018)

8. K.R. Karimullin et al., Bull. Russ. Acad. Sci. Phys. 82, 1478 (2018)

9. K.R. Karimullin et al., Bull. Russ. Acad. Sci. Phys. 81, 1396 (2017)

10. M.V. Knyazev et al., Phys. Stat. Sol. (RRL). 11, 1600414 (2017)

11. K. Karimullin et al., Meas. Sci. Technology. 24, 027002 (2013)

12. K.R. Karimullin et al., Bull. Russ. Acad. Sci. Phys. 78, 1254 (2014) 